\title{
A new procedure for synthesis of $\alpha$-aminophosphonates by aqueous formic acid as an effective and environment-friendly organocatalyst
}

\author{
ESMAEIL MOHAMMADIYAN ${ }^{\mathrm{a}}$, HOSSEIN GHAFURI ${ }^{\mathrm{b}, *}$ and ALI KAKANEJADIFARD ${ }^{\mathrm{a}}$ \\ ${ }^{a}$ Department of Chemistry, Faculty of science, University of Lorestan, Khorramabad, Iran \\ ${ }^{\mathrm{b}}$ Catalysis and Organic Synthesis Research Laboratory, Department of Chemistry, Iran University of Science \\ and Technology, Tehran, Iran \\ E-mail: ghafuri@iust.ac.ir
}

MS received 14 March 2017; revised 3 October 2017; accepted 3 October 2017; published online 20 November 2017

\begin{abstract}
Aqueous formic acid (37\%) as a green organocatalyst was used to synthesis of $\alpha$-aminophosphonates in one-pot, three-component Kabachnik-Fields reaction. The structures of compounds were determined by FTIR, ${ }^{1} \mathrm{H}-\mathrm{NMR}$ and ${ }^{13} \mathrm{C}$-NMR spectroscopy. After optimization of the experimental conditions, the reaction was carried out at $65^{\circ} \mathrm{C}$ under solvent- free condition. Use of a nontoxic effective organocatalyst, easy work up process and low-cost cleaning procedure are from the main advantages of this research.
\end{abstract}

Keywords. Organocatalyst; $\alpha$-aminophosphonate; formic acid; kabachnik-fields reaction; green chemistry.

\section{Introduction}

The $\alpha$-aminophosphonates and their derivatives are very useful compounds with wide range of applications in organic chemistry especially in medicinal chemistry. ${ }^{1}$ A large number of $\alpha$ - aminophosphonate derivatives are known as antiviral, ${ }^{2}$ antifungal, ${ }^{3}$ antibacterial ${ }^{4}$ and antitumor $^{5}$ agents. Thus, they form an important class of compounds with diverse biological activities (Figure 1).

Some other activities such as peptidomimetic, ${ }^{6}$ enzyme inhibitors, ${ }^{7}$ pharmacogenic agent, ${ }^{8}$ haptens of catalytic antibodies, ${ }^{9}$ inhibitors of UDP-galactopyranose mutase $^{10}$ and antitumor agents ${ }^{11-13}$ have been recognized for these compounds. Some of significant studies for synthesis of $\alpha$ - aminophosphonate derivatives are such as: synthesis of di or tri-alkyl phosphite derivatives, ${ }^{14}$ hydrogenation of aziridinylphosphonate, ${ }^{15}$ aldol-type reactions of (isocyanomethyl) phosphonates with aldehydes, ${ }^{16}$ addition of phosphites to sulphimines ${ }^{17}$ and catalyzed Mannich-type reaction. ${ }^{18}$ Among the versatile procedures, the Kabachnik - Fields reaction is one of the basic methods for preparation of $\alpha$-aminophosphonate which was discovered in 1952 independently by Kabachnik ${ }^{19}$ and Fields. ${ }^{20}$ Recently some new researches have been reported for promotion of one-pot Kabachnik-Fields reaction such as microwave irradiation, heating ${ }^{21}$ and acidic or basic catalysts. Some Lewis acid catalysts, such as $\mathrm{ZrOCl}_{2}$. $5 \mathrm{H}_{2} \mathrm{O},{ }^{22} \mathrm{Mg}\left(\mathrm{ClO}_{4}\right)_{2},{ }^{23} \mathrm{FeCl}_{3},{ }^{24} \mathrm{Al}\left(\mathrm{H}_{2} \mathrm{PO}_{4}\right),{ }^{25} \mathrm{BiCl}_{3},{ }^{26}$ $\mathrm{InCl}_{3},{ }^{27} \mathrm{YbCl}_{3},{ }^{28} \mathrm{In}(\mathrm{OTf})_{3},{ }^{29} \mathrm{Ce}(\mathrm{OTf})_{4},{ }^{30} \mathrm{Fe}_{3} \mathrm{O}_{4} @ \mathrm{ZrO}_{2}$ $/ \mathrm{SO}_{4}^{2-},{ }^{31} \mathrm{CAN},{ }^{32} \mathrm{TaCl}_{5} \mathrm{SiO}_{2},{ }^{33} \mathrm{SmI}_{2},{ }^{34} \mathrm{LiClO}_{4},{ }^{35}$ and some solid acids (montmorillonite KSF), silica sulphuric acid, and also some base catalysts like $\mathrm{CaCl}_{2}$, $\mathrm{PPh}_{3}$ and other catalysts such as $\mathrm{ZnO}, \mathrm{TiO}_{2}$, tosyl chloride and mesoporous aluminosilicate nanocage ${ }^{36}$ have been used to succeed this reaction. In spite of all researches, still there are some serious limitations such as hard work up process, long reaction time and expensive and toxic catalyst in these methods. With regard to importance of removal of toxic and hazardous catalysts from organic reactions, we decided to introduce formic acid as an efficient and green organocatalyst for synthesis of $\alpha$-aminophosphonates with interesting specifications in Kabachnik-Fields reaction. Formic acid is a colourless liquid with a pungent, penetrating odour and often used in an aqueous solution. Formica, is Latin word of ant and name of formic acid has been derived from its root referring to its early isolation by the distillation of ants' bodies. In nature, formic acid has been found in the stings and bites

\footnotetext{
*For correspondence

Electronic supplementary material: The online version of this article (https://doi.org/10.1007/s12039-017-1394-z) contains supplementary material, which is available to authorized users.
} 


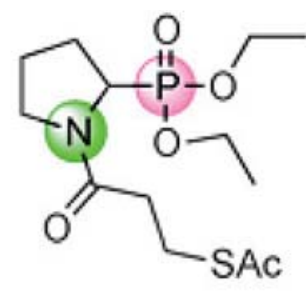

S-(3-(2-(diethoxyphosphoryl)pyrrolidin-1-yl)3-oxopropyl)ethanethioate

Anti-hypertensive agent

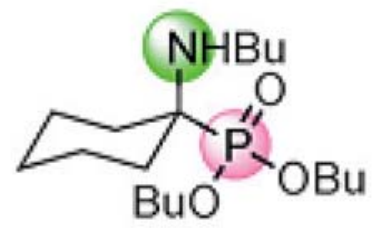

Dibutyl (1-(butylamino)cyclohexyl)phosphonate Herbicidal activity

Figure 1. $\alpha$-aminophosphonate with biological activities.

of many insects of the order Hymenoptera, including bees and ants. Furthermore formic acid is used as a preservative and antibacterial agent in livestock feed. Also it has been known as an important intermediate in chemical synthesis. In synthetic organic chemistry, it is used as a source of hydride ion which has been reported in some reactions like Eschweiler-Clarke and the Leuckart-Wallach. So its azeotrope with triethylamine is applied as a source of hydrogen in transfer hydrogenation. Sometime formic acid is employed as a volatile $\mathrm{pH}$ modifier in HPLC and capillary electrophoresis like acetic acid and trifluoroacetic acid. Also, formic acid can be a convenient source of carbon monoxide by being readily decomposed by sulphuric acid. Another important chemical activity of formic acid, is its useas reductant in combination with a catalyst, for the transfer hydrogenation of anilines ${ }^{37}$ and reduction of alkynes can selectively produce cis, transalkenes and alkanes, ${ }^{38} \alpha$-substituted acetophenones, ${ }^{39}$ $\beta$-keto esters ${ }^{40}$ and nitroarenes. In aspect of physical description it is a strong oxidizer, and with strong caustic properties. In our previous work, we showed formic acid as an efficient organocatalyst for synthesis of imines and $\alpha$-aminonitriles in Strecker reaction. ${ }^{41}$ In this work, in order to favour environmental considerations, we used aqueous formic acid as a green organocatalyst in the synthesis of $\alpha$-aminophosphonates through kabachnik field reaction.

\section{Experimental}

All of the chemicals were obtained from Merck and used without further purification. Infrared (IR) spectra were obtained on a Shimadzu FT-IR-8400S spectrophotometer using a $\mathrm{KBr}$ pellet. Melting points were measured by an Electro thermal 9100 apparatus. Analytical TLC was performed on Merck $0.2 \mathrm{~mm}$ silica gel $60 \mathrm{~F}-254 \mathrm{Al}$-plates. ${ }^{1} \mathrm{H}$ NMR and ${ }^{13} \mathrm{C}$ NMR spectra were recorded using Bruker DRX-500 Avance, Bruker DRX-400 Avance and Bruker DRX-250 Avance spectrometers at ambient temperature, respectively.

\subsection{General procedure for the synthesis of $\alpha$-aminophosphonate}

For synthesis of $\alpha$-aminophosphonate $1 \mathrm{c}$, in a $5 \mathrm{~mL}$ dry balloon, a mixture of $15 \mu \mathrm{L}$ catalyst (formic acid (37\%)) and $1 \mathrm{mmol}$ aldehyde was combined, after that $1 \mathrm{mmol}$ amine and $1.2 \mathrm{mmol}$ dimethylphosphite were added to the mixture. The reaction proceeds under solvent free condition and $65{ }^{\circ} \mathrm{C}$ temperature for a period of time on a vigorous magnetic stirrer. The progress of reaction by TLC in solvent samples 1: 1 hexane / ethyl acetate was followed. Finally, after completion of the reaction, the solid product was filtered, washed with deionized water. After recrystalization it was dried at room temperature.

\subsection{Spectral data of representative compounds}

2.2a Dimethyl [(phenyl) (phenylamino) methyl] phosphonate 1a: M.p.: $90-92{ }^{\circ} \mathrm{C},{ }^{1} \mathrm{HNMR}\left(400 \mathrm{MHz}, \mathrm{CDCl}_{3}\right)$ : $\delta 3.67(\mathrm{~d}, J=10.8 \mathrm{~Hz}, 3 \mathrm{H}), 3.89(\mathrm{~d}, J=11.2 \mathrm{~Hz}, 3 \mathrm{H})$, $5.86(\mathrm{~m}, 1 \mathrm{H}), 5.93(\mathrm{~d}, 1 \mathrm{H}), 7.28-8.09(\mathrm{~m}, 7 \mathrm{H}) \mathrm{ppm} .{ }^{13} \mathrm{C}$ $\mathrm{NMR}\left(\mathrm{CDCl}_{3}, 100 \mathrm{MHz}\right): \delta=53.70\left(\mathrm{~d},{ }^{2} J_{\mathrm{P}, \mathrm{C}}=6.7 \mathrm{~Hz}\right)$, $54.4\left(\mathrm{~d},{ }^{2} J_{\mathrm{P}, \mathrm{C}}=7.6 \mathrm{~Hz}\right), 54.7\left(\mathrm{~d},{ }^{1} J_{\mathrm{P}, \mathrm{C}}=152.0 \mathrm{~Hz}\right), 114.3$, 118.7, 123.0, 128.1, $129.0\left(\mathrm{~d},{ }^{2} J_{\mathrm{P}, \mathrm{C}}=4.4 \mathrm{~Hz}\right), 131.0,134.7$ $\left(\mathrm{d},{ }^{2} J_{\mathrm{P}, \mathrm{C}}=2.5 \mathrm{~Hz}\right), 146.6\left(\mathrm{~d},{ }^{2} J_{\mathrm{P}, \mathrm{C}}=14.9 \mathrm{~Hz}\right) \mathrm{ppm}$.

\section{2b Dimethyl[(2-chlorophenyl)(phenylamino)}

methyl]phosphonate 1b: M.p.: $128-129^{\circ} \mathrm{C}$, FT-IR (KBr, $\left.\nu_{\max } \mathrm{cm}^{-1}\right) ; 3311(\mathrm{~N}-\mathrm{H}), 1602,1519,1232,1033 ;{ }^{1} \mathrm{H}$ NMR $\left(\mathrm{CDCl}_{3}, 500 \mathrm{MHz}\right)=3.4(\mathrm{~d}, J=10.4 \mathrm{~Hz}, 3 \mathrm{H}), 3.8(\mathrm{~d}$, $J=10.7 \mathrm{~Hz}, 3 \mathrm{H}), 5.0(\mathrm{br}, \mathrm{NH}, 1 \mathrm{H}), 5.36(\mathrm{~d}, J=24.6 \mathrm{~Hz}$, $1 \mathrm{H}), 6.6\left(\mathrm{~d}, J=7.6(\mathrm{~m}, 9 \mathrm{H}) .{ }^{13} \mathrm{C}\right.$ NMR $\left(\mathrm{CDCl}_{3}, 125 \mathrm{MHz}\right)$ : 51. 04, 52.26, $54.24(\mathrm{~m}), 114$. 02, 119.13, 127.87, 129.39, $129.72,130.05,134.18,134.41\left(\mathrm{~d},{ }^{2} J_{\mathrm{P}, \mathrm{C}}=7.12 \mathrm{~Hz}\right), 145.87$ $\left(\mathrm{d},{ }^{2} J_{\mathrm{P}, \mathrm{C}}=14.7 \mathrm{~Hz}\right)$.

2.2c Dimethyl [(4-chlorophenyl) (phenylamino) methyl] phosphate 1c: M.p.: $139-140{ }^{\circ} \mathrm{C}, \mathrm{IR}\left(\mathrm{KBr}, \nu_{\max }\right.$ $\left.\mathrm{cm}^{-1}\right): 3319(\mathrm{~N}-\mathrm{H}), 1602,1494,1232,1033 ;{ }^{1} \mathrm{H}$ NMR (500 $\left.\mathrm{MHz}, \mathrm{CDCl}_{3}\right): \delta 3.55(\mathrm{~d}, J=10.8 \mathrm{~Hz}, 3 \mathrm{H}), 3.79(\mathrm{~d}, J=$ $10.5 \mathrm{~Hz}, 3 \mathrm{H}), 4.98\left(\mathrm{~d},{ }^{1} J_{\mathrm{P}-\mathrm{H}}=24 \mathrm{~Hz}, 1 \mathrm{H}\right), 7.3-8.2(\mathrm{~m}, 9 \mathrm{H})$.

${ }^{13} \mathrm{C}$ NMR $\left(125 \mathrm{MHz}, \mathrm{CDCl}_{3}\right): \delta=53.8,54.2,56.1,114.3$, 
$126.8,128.2\left(\mathrm{~d},{ }^{3} J_{\mathrm{p}-\mathrm{c}}=5.5 \mathrm{~Hz}\right), 128.4\left(\mathrm{~d},{ }^{3} J_{\mathrm{p}-\mathrm{c}}=3.0 \mathrm{~Hz}\right)$, $131.1,132.2,141.0,146.6\left(\mathrm{~d},{ }^{2} J_{\mathrm{p}-\mathrm{c}}=14.5 \mathrm{~Hz}\right) \mathrm{ppm}$.

2.2d Dimethyl (4-Dimethyl amino phenyl) (N-phenylamino) methylphosphonate 1d:: M.p.: $144^{\circ} \mathrm{C}$; IR (KBr, $\left.\nu_{\max } \mathrm{cm}^{-1}\right)$; 3446, 2926, 1350, 1251, 1167, 1030. ${ }^{1} \mathrm{H}$ NMR $\left(\mathrm{CDCl}_{3}, 400 \mathrm{MHz}\right): \delta=2.12(\mathrm{~s}, 1 \mathrm{H}), 2.93(\mathrm{~s}, 6 \mathrm{H}), 3.51(\mathrm{~d}$, $J=10.4 \mathrm{~Hz}, 3 \mathrm{H}), 3.78(\mathrm{~d}, J=10.4 \mathrm{~Hz}, 3 \mathrm{H}), 4.70(\mathrm{~d}$, $\left.{ }^{1} J_{\mathrm{P}-\mathrm{H}}=23.6 \mathrm{~Hz}, 2 \mathrm{H}\right), 6.63(\mathrm{~d}, \mathrm{~d}, J=8.6 \mathrm{~Hz}, J=0.8 \mathrm{~Hz}$, $2 \mathrm{H}), 6.68(\mathrm{~m}, 3 \mathrm{H}), 7.12(\mathrm{~m}, 2 \mathrm{H}), 7.32(\mathrm{t}, \mathrm{t} J=6.8 \mathrm{~Hz}$, $J=2 \mathrm{~Hz}, 2 \mathrm{H}) \mathrm{ppm}$.

2.2e Dimethyl(4- methoxy phenyl)(N-phenylamino) methylphosphonate 1e: M.p.: $123-124^{\circ} \mathrm{C}$; IR $\left(\mathrm{KBr}, \nu_{\max }\right.$ $\left.\mathrm{cm}^{-1}\right): 3290,1602,1508,1240,1024 \mathrm{~cm}^{-1} ; 1 \mathrm{H} \mathrm{NMR}$ $\left(\mathrm{CDCl}_{3}, 400 \mathrm{MHz}\right): 2.93$ (s $\left.3 \mathrm{H}\right), 3.50(3 \mathrm{H}, \mathrm{d}, J=10.4 \mathrm{~Hz})$, $3.77(\mathrm{~d}, J=10.8 \mathrm{~Hz} 3 \mathrm{H}), 4.74\left(1 \mathrm{H}, \mathrm{d},{ }^{1} J_{\mathrm{P}-\mathrm{H}}=24.08 \mathrm{~Hz}\right)$, $6.60(\mathrm{~d}, \mathrm{~d}, J=8.6 \mathrm{~Hz}, J=1.2 \mathrm{~Hz}, 2 \mathrm{H}), 6.72(\mathrm{t}, J=7.2 \mathrm{~Hz}$ $1 \mathrm{H}), 6.90(\mathrm{~d}, J=8.4 \mathrm{~Hz}, 2 \mathrm{H}), 7.13(\mathrm{t}, J=8.2 \mathrm{~Hz}, 2 \mathrm{H}), 7.40(\mathrm{t}$, $\mathrm{t}, J=2.4 \mathrm{~Hz}, 2.4 \mathrm{~Hz}, 2 \mathrm{H}), \mathrm{ppm} ;{ }^{13} \mathrm{C} \mathrm{NMR}\left(\mathrm{CDCl}_{3}, 100\right.$ MHz): 54.01, 55.82, 57.52, 115.05, 115.74, 120.05, 128.87, $129.45,129.73,146.60,146.90,159.96 \mathrm{ppm}$.

2.2f Dimethyl(4-methylphenyl)(N-phenylamino)methylphosphonate 1f: M.p.: $128^{\circ} \mathrm{C}$; IR $\left(\mathrm{KBr}, \nu_{\max } \mathrm{cm}^{-1}\right)$ : 3313, 1602, 1498, 1232, $1031 \mathrm{~cm}^{-1}$; ${ }^{1} \mathrm{H}$ NMR(400 MHz, $\left.\mathrm{CDCl}_{3}\right): \delta 2.18(\mathrm{~s}, 3 \mathrm{H}), 3.49(\mathrm{~d}, J=10.4 \mathrm{~Hz}, 3 \mathrm{H}), 3.79(\mathrm{~d}$, $J=10.8 \mathrm{~Hz}, 3 \mathrm{H}), 4.82\left(\mathrm{~d},{ }^{1} J_{\mathrm{P}-\mathrm{H}}=23.6 \mathrm{~Hz}, 1 \mathrm{H}\right), 6.60(\mathrm{~d}, \mathrm{~d}$ $J=8.6 \mathrm{~Hz}, J=1.2 \mathrm{~Hz}, 2 \mathrm{H}), 6.71(\mathrm{t}, J=7.2 \mathrm{~Hz}, 1 \mathrm{H}), 6.90$ $(\mathrm{d}, J=8.4 \mathrm{~Hz}, 2 \mathrm{H}), 7.12(\mathrm{t}, J=7.8 \mathrm{~Hz}, 2 \mathrm{H}), 7.40(\mathrm{t}, \mathrm{t}$, $J=6.4 \mathrm{~Hz}, J=2.4 \mathrm{~Hz} 2 \mathrm{H}$ ) ppm.

$2.2 \mathrm{~g}$ Dimethyl(Terephthal)(N-phenylamino)methylphosphonate 1g: M.p.: $130-135^{\circ} \mathrm{C}$; $\mathrm{IR}\left(\mathrm{KBr}, \nu_{\max } \mathrm{cm}^{-1}\right)$ : 3290, 1602, 1508, 1240, $1024 \mathrm{~cm}^{-1} ;{ }^{1} \mathrm{H} \mathrm{NMR}\left(\mathrm{CDCl}_{3}, 400\right.$ MHz): 2.83 (br, s $1 \mathrm{H}),(3.51(3 \mathrm{H}, \mathrm{d}, J=10.4 \mathrm{~Hz}), 3.77$ $(\mathrm{s}, 3 \mathrm{H}), 3.80(\mathrm{~d}, J=1.2 \mathrm{~Hz}, 3 \mathrm{H}), 4.77\left(1 \mathrm{H}, \mathrm{d},{ }^{1} J_{\mathrm{P}-\mathrm{H}}=\right.$ 24.08 Hz). 6.60-7.41 (9H, m) ppm; ${ }^{13} \mathrm{C} \mathrm{NMR}\left(\mathrm{CDCl}_{3}, 100\right.$ MHz):54.01, 55.82, 57.52, 115.05, 115.74, 120.05, 128.87, $129.45,129.73,146.60,146.90,159.96 \mathrm{ppm}$.

2.2h Dimethyl(4-chlorophenyl)(N-4-nitrophenyla-mino) methylphosphonate 1J: M.p.: $160-162{ }^{\circ} \mathrm{C}$; IR $\left(\mathrm{KBr}, \nu_{\max }\right.$ $\mathrm{cm}^{-1}$ ): 3413(N-H), 3176(br O-H), 1602, 1504, 1231, 1029; ${ }^{1} \mathrm{H}$ NMR $\left(500 \mathrm{MHz}, \mathrm{CDCl}_{3}\right): \delta 3.45(\mathrm{~d}, J=10.5 \mathrm{~Hz}, 3 \mathrm{H})$, $3.74(\mathrm{~d}, J=10.7 \mathrm{~Hz}, 3 \mathrm{H}), 4.73\left(\mathrm{~d},{ }^{1} J_{\mathrm{P}-\mathrm{H}}=23.8 \mathrm{~Hz}, 1 \mathrm{H}\right)$, $5.82($ br $2 \mathrm{H}) 6.60(\mathrm{~d}, J=7.5 \mathrm{~Hz}, 2 \mathrm{H}), 6.70(\mathrm{t}, J=7.2 \mathrm{~Hz}$, $1 \mathrm{H}), 6.79(\mathrm{~d}, J=8.0 \mathrm{~Hz}, 1 \mathrm{H}), 6.91(\mathrm{~d}, J=6.51 \mathrm{~Hz} 1 \mathrm{H}), 7.07$ $(\mathrm{d}, J=7.6 \mathrm{~Hz}, 2 \mathrm{H}), 7.17(\mathrm{~m}, 2 \mathrm{H}) \mathrm{ppm} .{ }^{13} \mathrm{C} \mathrm{NMR}\left(\mathrm{CDCl}_{3}\right.$, $125 \mathrm{MHz}): 54.0,54.1,54.7-56.3\left(\mathrm{~d},{ }^{1} J_{\mathrm{c}-\mathrm{p}}=152 \mathrm{~Hz}\right), 113.9$, 114.4, 115.8, 118.7, 119.7, 129.2, 129.9, 136.7, 145.9, 146.0, $157.3 \mathrm{ppm}$.

2.2i Dimethyl(2,6-dichlorophenyl)(4-nitrophenylamino)methylphosphonate $1 \mathrm{k}$ : M.p.: $135^{\circ} \mathrm{C}$; IR $(\mathrm{KBr}$, $\left.v_{\max } \mathrm{cm}^{-1}\right): 3303,2952,1602,1498,1315,1240,1180$, $1051,1029 \mathrm{~cm}^{-1} ;{ }^{1} \mathrm{H}$ NMR $\left(\mathrm{CDCl}_{3}, 400 \mathrm{MHz}\right): \delta=3.65$ (d, $J=10.8 \mathrm{~Hz}, 3 \mathrm{H}), 3.90(\mathrm{~d}, J=11.2 \mathrm{~Hz}, 3 \mathrm{H}), 5.90(\mathrm{~d}$ $\mathrm{d}, J=9.2 \mathrm{~Hz}, J=30.8 \mathrm{~Hz} 2 \mathrm{H}), 5.87(\mathrm{~s} \mathrm{br}, 1 \mathrm{H}), 6.62(\mathrm{~d}$, $J=9.2 \mathrm{~Hz}, 2 \mathrm{H}) 7.22(\mathrm{t}, \mathrm{d}, J=8 \mathrm{~Hz}, J=2 \mathrm{~Hz} 1 \mathrm{H}), 7.30(\mathrm{~d}$, $J=1.2 \mathrm{~Hz}, 1 \mathrm{H}) 7.40(\mathrm{~d}, \mathrm{t}, J=8 \mathrm{~Hz}, J=1.2 \mathrm{~Hz}, 1 \mathrm{H}), 8.1$ $(\mathrm{d}, J=9.2 \mathrm{~Hz}, 2 \mathrm{H}) \mathrm{ppm}$.

\section{2j Dimethyl(4-nitrophenyl)(4-nitrophenylamino)}

methylphosphonate 11: M.p.: $123^{\circ} \mathrm{C}$; IR ( $\mathrm{KBr}, \nu_{\max }$ $\left.\mathrm{cm}^{-1}\right): 3310,1602,1498,1237,1027 \mathrm{~cm}^{-1} ;{ }^{1} \mathrm{H}$ NMR $(500$ $\left.\mathrm{MHz}, \mathrm{CDCl}_{3}\right): \delta 3.18(\mathrm{~d}, J=10.5 \mathrm{~Hz}, 3 \mathrm{H}), 3.85(\mathrm{~d}, J=$ $10.6 \mathrm{~Hz}, 3 \mathrm{H}), 5.69(\mathrm{~d}, J=24.0,1 \mathrm{H}), 6.58(\mathrm{~d}, J=8.0 \mathrm{~Hz}$, $2 \mathrm{H}), 6.69(\mathrm{t}, J=7.6 \mathrm{~Hz}, 1 \mathrm{H}), 7.06(\mathrm{t}, J=7.7 \mathrm{~Hz}, 2 \mathrm{H}), 7.47$ (t, $7.7 \mathrm{~Hz}, 1 \mathrm{H}), 7.57(\mathrm{t}, J=7.3 \mathrm{~Hz}, 1 \mathrm{H}), 7.65(\mathrm{t}, 7.8 \mathrm{~Hz}, 1 \mathrm{H})$, $7.83(\mathrm{~d}, J=7.3 \mathrm{~Hz}, 2 \mathrm{H}), 7.94(\mathrm{~d}, J=8.1 \mathrm{~Hz}, 1 \mathrm{H}), 8.26$ $(\mathrm{d}, J=8.7 \mathrm{~Hz}, 1 \mathrm{H}) \mathrm{ppm} .{ }^{13} \mathrm{C} \mathrm{NMR}\left(\mathrm{CDCl}_{3}, 125 \mathrm{MHz}\right)$ : $\delta=50.96,52.18,54.13(\mathrm{~d}, J=7.1 \mathrm{~Hz}), 114.04,118.89$, $123.03,125.96(\mathrm{~m}), 126.95,129.10\left(\mathrm{~d} .{ }^{3} J_{\mathrm{C}-\mathrm{P}}=3.6 \mathrm{~Hz}\right)$, $129.55,129.66,131.79\left(\mathrm{~d},{ }^{3} J_{\mathrm{C}-\mathrm{P}}=4.5 \mathrm{~Hz}\right), 134.30,146.30$ $\left(\mathrm{d},{ }^{2} J_{\mathrm{C}-\mathrm{P}}=14.1 \mathrm{~Hz}\right) \mathrm{ppm}$.

2.2k Dimethyl (Terephthal) (N-4-nitrophenylamino) methylphosphonate $\mathbf{1 m}$ : M.p.: $237^{\circ} \mathrm{C}$, IR $\left(\mathrm{KBr}, v_{\max }\right.$ $\left.\mathrm{cm}^{-1}\right)$; 3446, 2926, 1350, 1251, 1167, 1030. ${ }^{1} \mathrm{H}$ NMR (DMSO, $250 \mathrm{MHz}): \delta=3.45(\mathrm{~m}, 6 \mathrm{H}) 3.70(\mathrm{~m}, 6 \mathrm{H}), 4.90(\mathrm{~s}$ br), $5.05(\mathrm{dd}, J=23.5 \mathrm{~Hz}, J=5.2 \mathrm{~Hz} 2 \mathrm{H}), 6.70(\mathrm{~m}, 4 \mathrm{H})$, $7.39(\mathrm{~m}, 6 \mathrm{H}), 7.94(\mathrm{~d}, J=8.5 \mathrm{~Hz} 4 \mathrm{H}) \mathrm{ppm}$.

2.21 Dimethyl (4-methylphenyl) (N-4-nitrophenylamino) methylphosphonate 1n: M.p.: $158^{\circ} \mathrm{C}$; IR (KBr, $\nu_{\max } \mathrm{cm}^{-1}$ ) 3306, 1600, 1500, 1313, 1240, 1027. ${ }^{1} \mathrm{H}$ NMR $\left(400 \mathrm{MHz}, \mathrm{CDCl}_{3} \mathrm{~d}_{6}\right): \delta=2.34(\mathrm{~s}, 3 \mathrm{H}), 3.47(\mathrm{~d}, J=10.8 \mathrm{~Hz}$, $2 \mathrm{H}), 3.8(\mathrm{~d}, J=11.2,3 \mathrm{H}), 4.83(\mathrm{~d}, J=24 \mathrm{~Hz}, 3 \mathrm{H}), 5.9(\mathrm{~s}, \mathrm{br}$ $1 \mathrm{H}), 6.6(\mathrm{~d}, J=9.2 \mathrm{~Hz}, 2 \mathrm{H}), 7.2(\mathrm{~d}, J=8.0 \mathrm{~Hz}, 2 \mathrm{H}), 7.35$ $(\mathrm{d} \mathrm{d}, J=12.4 \mathrm{~Hz}, J=2.0 \mathrm{~Hz}, 2 \mathrm{H}) 8.0(\mathrm{~d}, J=10.8 \mathrm{~Hz}, 2 \mathrm{H})$ ppm.

2.2m Dimethyl (3-hydroxyphenyl) (N-4-nitrophenylamino)methylphosphonate 1p: M.p.: $165^{\circ} \mathrm{C}$, IR ( $\mathrm{KBr}$, $\nu_{\max } \mathrm{cm}^{-1}$ ) 3301, 2950, 1612, 1514, 1458, 1337, 1238, $1178,1058,1027 .{ }^{1} \mathrm{H} \mathrm{NMR}\left(\mathrm{CDCl}_{3}, 250 \mathrm{MHz}\right): \delta 3.41(\mathrm{~d}$, $J=10.5 \mathrm{~Hz}, 3 \mathrm{H}), 3.68(\mathrm{~d}, J=14.2 \mathrm{~Hz}, 3 \mathrm{H}), 4.71(\mathrm{~d}$, $J=23.7,1 \mathrm{H}), 5.49(\mathrm{~s}, \mathrm{br}, 1 \mathrm{H}), 6.52(\mathrm{~d}, J=3.2 \mathrm{~Hz}, 1 \mathrm{H})$, $6.76(\mathrm{~d}, J=7.7 \mathrm{~Hz}, 1 \mathrm{H}), 6.9(\mathrm{~m}, 2 \mathrm{H}), 7.14(\mathrm{t}, J=7.7 \mathrm{~Hz}$, 1H) $7.96(\mathrm{~d}, J=7.2 \mathrm{~Hz}, 1 \mathrm{H}) \mathrm{ppm}$.

2.2n Dimethyl(4-hydroxyphenyl)(N-4-nitrophenylamino)methylphosphonate 1q: M.p.: $166^{\circ} \mathrm{C}$, IR ( $\mathrm{KBr}$, $\nu_{\max } \mathrm{cm}^{-1}$ ) 3298, 3074, 2921, 2852, 2432, 1600, 1546, 1490, 1328, 1278, 1234, 1178, 1112, 1091, 1051, 1024, ${ }^{1} \mathrm{H}$ NMR $\left(\mathrm{CDCl}_{3}, 250 \mathrm{MHz}\right): \delta 3.4(\mathrm{~d}, J=10.5 \mathrm{~Hz}, 3 \mathrm{H}), 3.70(\mathrm{~d}$, $J=10.7 \mathrm{~Hz}, 3 \mathrm{H}), 4.74\left(\mathrm{~d},{ }^{1} J_{\mathrm{P}-\mathrm{H}}=23.5 \mathrm{~Hz}, 1 \mathrm{H}\right), 5.6(\mathrm{~s}$, $2 \mathrm{H}), 6.52(\mathrm{~d}, J=9 \mathrm{~Hz}, 2 \mathrm{H}), 6.80(\mathrm{~d}, J=8.5 \mathrm{~Hz}, 2 \mathrm{H}) 7.20$ $(\mathrm{d}, J=9.4,2 \mathrm{H}), 7.96(\mathrm{~d}, J=9.2 \mathrm{~Hz}, 2 \mathrm{H}) \mathrm{ppm}$.

2.2o Dimethyl(2-chlorophenyl)(4-methylphenyla-mino) methylphosphonate 1r: M.p.: $158^{\circ} \mathrm{C}$, IR $\left(\mathrm{KBr}, v_{\max }\right.$ $\mathrm{cm}^{-1}$ ) 3271, 3070, 2954, 2923, 2848, 1483, 1182. ${ }^{1} \mathrm{H}$ NMR 


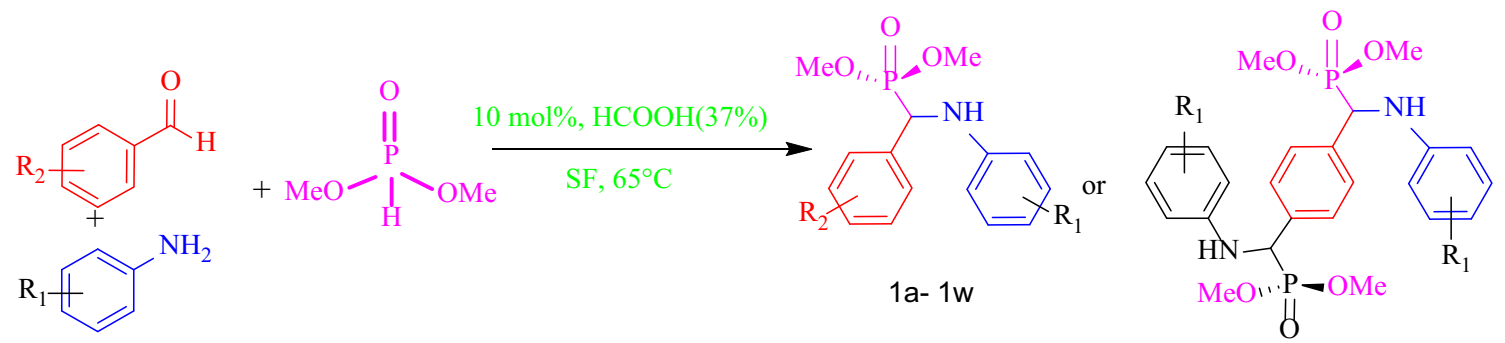

Scheme 1. Kabachnik-Fields reaction by aqueous formic acid as organocatalyst.

Table 1. Optimization of reaction conditions for the synthesis of $\alpha$ aminophosphonate by aqueous formic acid as green organocatalyst. ${ }^{\text {a }}$

\begin{tabular}{lccccc}
\hline Entry & Temp. $\left({ }^{\circ} \mathrm{C}\right)$ & Solvent & Time $(\mathrm{min})$ & Catalyst(mL) & Yield (\%) \\
\hline 1 & 25 & - & $2 \mathrm{~h}$ & 0 & Trace \\
2 & 25 & - & 25 & 15 & 45 \\
3 & 40 & - & 25 & 10 & 50 \\
4 & 65 & - & 25 & 10 & 85 \\
5 & 80 & - & 25 & 10 & 80 \\
6 & 65 & $\mathrm{H}_{2} \mathrm{O}$ & 25 & 10 & - \\
7 & 65 & EtOH & 25 & 10 & 80 \\
8 & 65 & Toluene & 25 & 10 & 80 \\
9 & 65 & n-Hexane & 25 & 10 & 83 \\
10 & 65 & - & 25 & 15 & 60 \\
11 & 65 & - & 25 & 20 & 50 \\
12 & 65 & - & 25 & 25 & 55 \\
\hline
\end{tabular}

a $1 \mathrm{mmol}$ aldehyde, $1 \mathrm{mmol}$ amine and $1.2 \mathrm{mmol}$ dimethylphosphate.

$\left(\mathrm{CDCl}_{3}, 250 \mathrm{MHz}\right): \delta=2.20(\mathrm{~s}, 3 \mathrm{H}), 3.46(\mathrm{~d}, J=10.5 \mathrm{~Hz}$, $3 \mathrm{H}), 3.70(\mathrm{~d}, J=11 \mathrm{~Hz}, 3 \mathrm{H}), 3.71(\mathrm{~s}, 3 \mathrm{H}), 5.40(\mathrm{~d}$, $\left.{ }^{1} J_{\mathrm{P}-\mathrm{H}}=24.7 \mathrm{~Hz}, 1 \mathrm{H}\right), 5.86(\mathrm{~s}$, br $1 \mathrm{H}), 6.53(\mathrm{~d}, J=8.3 \mathrm{~Hz}$, $2 \mathrm{H}), 6.92(\mathrm{~d}, J=8.3 \mathrm{~Hz}, 2 \mathrm{H}), 7.25(\mathrm{~m}, 2 \mathrm{H}), 7.40(\mathrm{~d}$, $J=8.2 \mathrm{~Hz}, 1 \mathrm{H}), 7.60(\mathrm{~d}, J=8.2 \mathrm{~Hz}, 1 \mathrm{H}) .{ }^{13} \mathrm{C} \mathrm{NMR}$ $\left(\mathrm{CDCl}_{3}, 62.9 \mathrm{MHz}\right): \delta 20.3,50.1,52.5,53.8(\mathrm{~m}), 113.7,126.8$, 127.4 (d), 127.9, 128.8 (d), 129.2 (d), 129.5 (d), 129.8 ppm.

2.2p Dimethyl[(4-nitrophenyl)-(N-2-methylphenylamino)methyl]phosphonate 1t: M.p.: $146-150^{\circ} \mathrm{C}$; IR $\left(\mathrm{KBr}, v_{\max } \mathrm{cm}^{-1}\right): 3331,1602,1498,1449,1230,1028 \mathrm{~cm}^{-1}$; ${ }^{1} \mathrm{H} \mathrm{NMR}\left(250 \mathrm{MHz}, \mathrm{CDCl}_{3}\right): \delta 2.19(\mathrm{~s}, 3 \mathrm{H}), 3.70(\mathrm{~d}, J==$ $8.0 \mathrm{~Hz}, 2 \mathrm{H}) 7.67(\mathrm{~d}, J=8.5 \mathrm{~Hz}, 2 \mathrm{H}), 8.2(\mathrm{~d}, J=8.2 \mathrm{~Hz}$, $2 \mathrm{H}) ;{ }^{13} \mathrm{C} \mathrm{NMR}\left(\mathrm{CDCl}_{3}, 62.9 \mathrm{MHz}\right): \delta 20.3,54.2(\mathrm{~m}), 57.0(\mathrm{~d})$, 114.0(d), 123.8(d), 123.9, 128.6(d), 129.8, 142.9 (d), 143.7 (d), 147.8(d) ppm.

\section{Results and Discussion}

Aqueous formic acid was used to synthesis of $\alpha$ aminophosphonate by a one-pot, three-component reaction of aldehyde, amine and dimethylphosphite under solvent-free conditions (Scheme 1).

\subsection{Optimization of synthetic conditions for}

kabachnik-fields reaction catalyzed by aqueous formic acid

To determine the best experimental conditions, the reaction of, 4-chlorobenzaldehyde, aniline and dimethylphosphite was considered as the model of reaction (Table 1).

For optimization of the best condition to carry out the reaction different conditions were tested and the results summarized in Table 1.

Positive effect of aqueous formic acid in promotion of this reaction has been indicated in Table 1. Without using catalyst, No significant amount of product is obtained after $2 \mathrm{~h}$. To determine the optimum amount of catalyst, we compared four diverse amounts of catalyst and the results show that $10 \%$ is the best amount for this reaction. More or lower than this range, can decrease the yield percentage. To determination the best solvent condition, some current solvent were tested and compared with solvent free (SF) condition and SF had shown the best result in this reaction. In aspect of temperature conditions, among the various temperatures, the best result was obtained at $65^{\circ} \mathrm{C}$ (Table 2). 
Table 2. Synthesized derivatives of $\alpha$-aminophosphonate in the presence of aqueous formic acid as reaction organocatalyst ${ }^{\mathrm{a}}$.

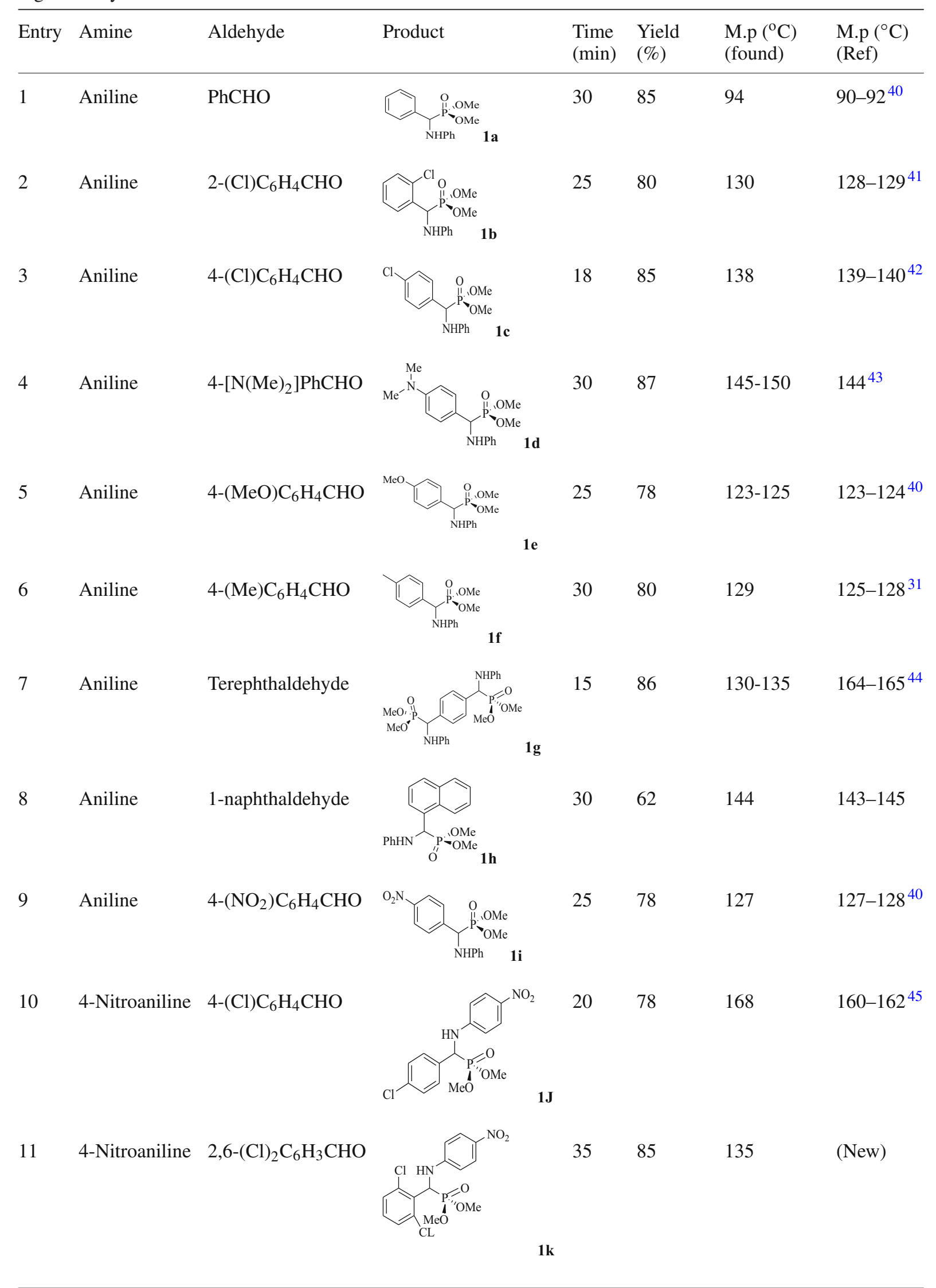


Table 2. (contd.)

\begin{tabular}{|c|c|c|c|c|c|c|c|}
\hline Entry & Amine & Aldehyde & Product & $\begin{array}{l}\text { Time } \\
(\min )\end{array}$ & $\begin{array}{l}\text { Yield } \\
(\%)\end{array}$ & $\begin{array}{l}\text { M.p }\left({ }^{\circ} \mathrm{C}\right) \\
\text { (found) }\end{array}$ & $\begin{array}{l}\text { M.p }\left({ }^{\circ} \mathrm{C}\right) \\
\text { (Ref) }\end{array}$ \\
\hline 12 & 4-Nitroaniline & $4-\left(\mathrm{NO}_{2}\right) \mathrm{C}_{6} \mathrm{H}_{4} \mathrm{CHO}$ & & 25 & 70 & 123 & $186^{46}$ \\
\hline 13 & 4-Nitroaniline & Terephthaldehyde & & 15 & 80 & 237 & (New) \\
\hline 14 & 4-Nitroaniline & 4-(Me) $\mathrm{C}_{6} \mathrm{H}_{4} \mathrm{CHO}$ & & 45 & 80 & 158 & (New) \\
\hline
\end{tabular}

15 4-Nitroaniline 4-(OMe) $\mathrm{C}_{6} \mathrm{H}_{4} \mathrm{CHO}$

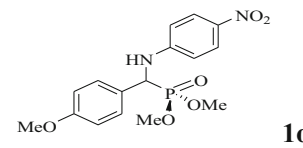

16 4-Nitroaniline $3-(\mathrm{OH}) \mathrm{C}_{6} \mathrm{H}_{4} \mathrm{CHO}$

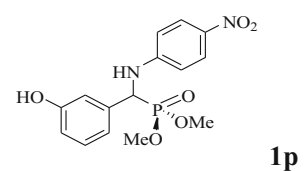

(New)

$1 p$

17 4-Nitroaniline 4- $(\mathrm{OH}) \mathrm{C}_{6} \mathrm{H}_{4} \mathrm{CHO}$

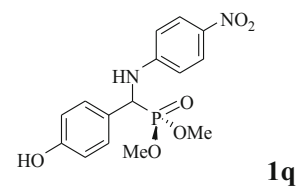

$\begin{array}{llll}30 & 68 & 166 & (\mathrm{New})\end{array}$

19

$18 p$-Toluidine $2-(\mathrm{Cl}) \mathrm{C}_{6} \mathrm{H}_{4} \mathrm{CHO}$

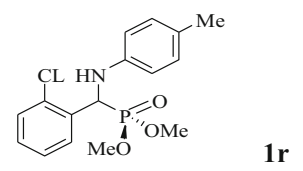

$80 \quad 158 \quad(\mathrm{New})$

$19 p$-Toluidine $\quad 4-(\mathrm{Cl}) \mathrm{C}_{6} \mathrm{H}_{4} \mathrm{CHO}$

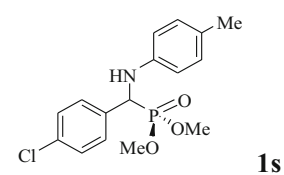

$20 p$-Toluidine $4-\left(\mathrm{NO}_{2}\right) \mathrm{C}_{6} \mathrm{H}_{4} \mathrm{CHO}$

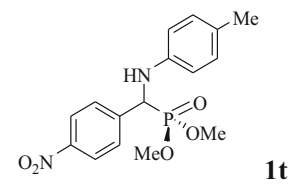


Table 2. (contd.)

\begin{tabular}{|c|c|c|c|c|c|c|c|}
\hline Entry & Amine & Aldehyde & Product & $\begin{array}{l}\text { Time } \\
(\mathrm{min})\end{array}$ & $\begin{array}{l}\text { Yield } \\
(\%)\end{array}$ & $\begin{array}{l}\text { M.p }\left({ }^{\circ} \mathrm{C}\right) \\
\text { (found) }\end{array}$ & $\begin{array}{l}\text { M.p }\left({ }^{\circ} \mathrm{C}\right) \\
\text { (Ref) }\end{array}$ \\
\hline 21 & $p$-Toluidine & $4-(\mathrm{MeO}) \mathrm{C}_{6} \mathrm{H}_{4} \mathrm{CHO}$ & & 35 & 75 & 90 & $96-99^{46}$ \\
\hline 22 & $p$-Toluidine & $\mathrm{PhCHO}$ & & 25 & 77 & 70 & $68-71^{46}$ \\
\hline 23 & 4-Bromoaniline & $\mathrm{PhCHO}$ & & 40 & 70 & 65 & $60^{47}$ \\
\hline
\end{tabular}

${ }^{a} 1 \mathrm{mmol}$ aldehyde, $1 \mathrm{mmol}$ amine and $1.2 \mathrm{mmol}$ dimethylphosphate, $15 \mu \mathrm{L}$ catalyst (formic acid $(37 \%)$ ), $65{ }^{\circ} \mathrm{C}$ temperature.

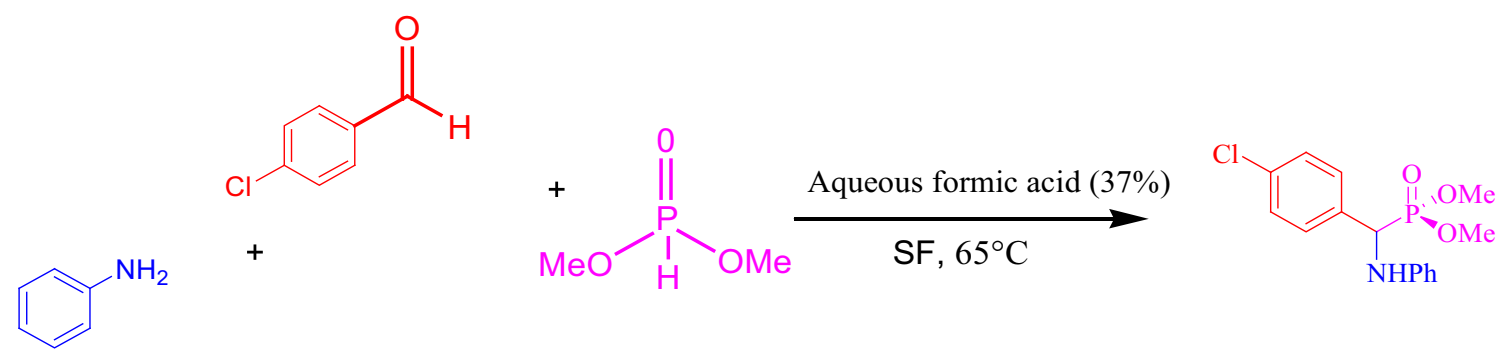

Scheme 2. The reaction of, 4-chlorobenzaldehyde, aniline and dimethylphosphite to preparation of $\alpha$ -aminophosphonates.

After optimization of the reaction condition, in order to generalize the method, it was expanded with versatile aldehydes and amines for synthesis of other derivatives of $\alpha$-aminophosphonates and the results were summarized in (Table 2). Six derivatives including (Entry 11, $13,14,16,17,18)$ have been synthesized for the first time in this work.

\subsection{The proposed mechanism of the}

Kabachnik-Fields reaction in the presence of aqueous formic acid

The suggested mechanism to synthesis of $\alpha$-aminophosphonate by aqueous formic acid as organocatalyst is shown in Scheme 3.

As shown in Scheme 3, the first step is the activation of carbonyl groups in aldehydes by hydrogen bond interaction with $\mathrm{HCOOH}$ (I). Second, a nucleophilic addition of amine to activated carbonyl, cause

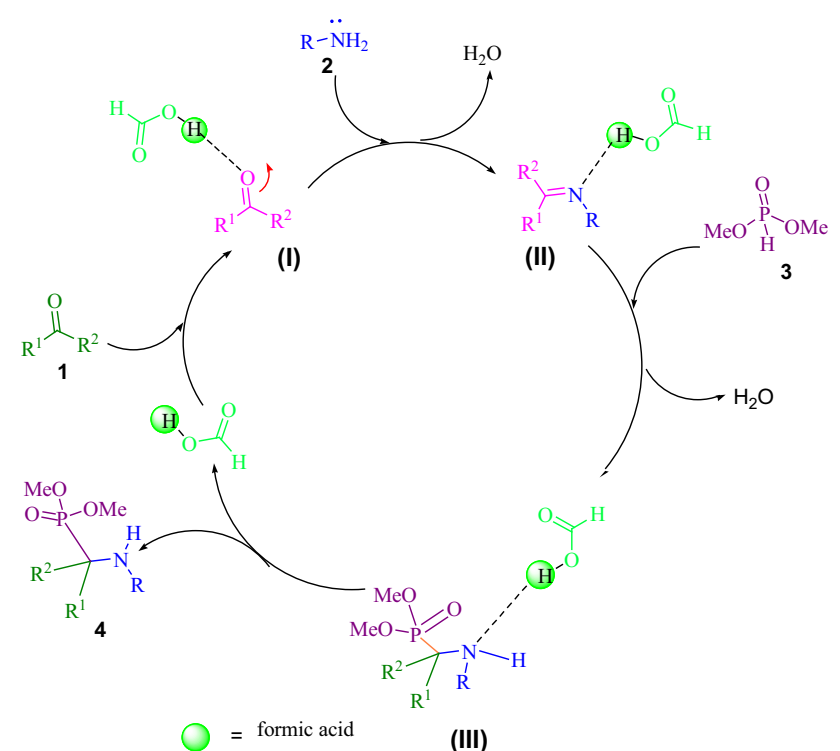

Scheme 3. The proposed mechanism of the KabachnikFields reaction by aqueous formic acid as organocatalyst. 
the formation of an imine intermediate (II). The formation of imine intermediate by formic acid was reported in our former research ${ }^{41}$. Also, the addition of nucleophilic phosphonate 3 to imine cause the formation of $\alpha$-aminophosphonate 4 . After the separation of catalyst, the pure product can be obtained.

\section{Conclusions}

Aqueous formic acid was demonstrated as a green and effective organocatalyst in Kabachnik-Fields reaction to synthesize $\alpha$-aminophosphonate derivatives. The ecofriendly and low cost organocatalysts and solvent free condition provide considerable advantages for this procedure. Also, this method has shown several benefits such as: easy work up process, short reaction time and lack of toxicity. Six of the reported derivatives were synthesized for the first time in this study.

\section{Supplementary Information (SI)}

Additional experimental data and spectroscopic characterization data are given in the Supplementary Information. Supplementary Information is available at www.ias.ac.in/ chemsci.

\section{Acknowledgements}

This work was supported by the Research Council of Department of Chemistry Iran University of Science.

\section{References}

1. Bartlett P A, Hanson J E and Giannousis P P 1990 Potent inhibition of pepsin and penicillopepsin by phosphoruscontaining peptide analogues J. Org. Chem. 556268

2. Xu Y, Yan K, Song B, Xu G, Yang S, Xue W, Hu D, Lu P, Ouyang G, Jin L and Chen Z 2006 Synthesis and antiviral bioactivities of $\alpha$-aminophosphonates containing alkoxyethyl moieties Molecules 11666

3. Ouimette D and Coffey M 1989 Comparative antifungal activity of four phosphonate compounds against isolates of nine Phytophthora species Phytopathology 79761

4. Kumar B S, Sankar A, Reddy C S, Nayak S and Raju C N 2007 Synthesis and antimicrobial activity of 2, 10-dichloro-6-substituted aminobenzyl-12H-dibenzo [d, g] [1, 3, 2] dioxaphosphocin-6-oxides Arkivoc 13155

5. Song B-A, Wu Y-L, Yang S, Hu D-Y, He X-Q and Jin L-H 2003 Synthesis and bioactivity of a-aminophosphonates containing fluorine Molecules 8186

6. Xia M and Lu Y-d 2007 Ultrasound-assisted one-pot approach to $\alpha$-amino phosphonates under solvent-free and catalyst-free conditions Ultrason. Sonochem. 14235

7. Kaboudin B and Sorbiun M $2007 \beta$-Cyclodextrin as an efficient catalyst for the one-pot synthesis of 1aminophosphonic esters in water Tetrahedron Lett. 48 9015
8. Baylis E K, Campbell C D and Dingwall J G 1Aminoalkylphosphonous acids Part 1 Isosteres of the protein amino acids J. Chem. Soc. Perkin.1 2845

9. Smith A B, Taylor C M, Benkovic S J and Hirschmann R 1994 Peptide bond formation via catalytic antibodies: Synthesis of a novel phosphonate diester hapten Tetrahedron Lett. 356853

10. Pan W, Ansiaux C and Vincent S P 2007 Synthesis of acyclic galactitol-and lyxitol-aminophosphonates as inhibitors of UDP-galactopyranose mutase Tetrahedron Lett. 484353

11. Bloemink M J, Diederen J J, Dorenbos J P, Heetebrij R J, Keppler B K and Reedijk J 1999 Calcium Ions Do Accelerate the DNA Binding of New Antitumor-Active Platinum Aminophosphonate Complexes Eur. J. Inorg. Chem. 19991655

12. He X-P, Li C, Jin X-P, Song Z, Zhang H-L, Zhu C-J, Shen Q, Zhang W, Sheng L, Shi X-X, Tang Y, Li J, Chen G-R and Xie J 2011 Microwave-assisted construction of triazole-linked amino acid-glucoside conjugates as novel PTP1B inhibitors New J. Chem. 35622

13. Rao X, Song Z, Yao X, Gao H and Ye B 2008 The green approaches for the synthesis of N-dehydroabietic $\alpha$-aminophosphonates Nat. Prod. Res. 22890

14. Gautier I, Ratovelomanana-Vidal V, Savignac P and Genêt J-P 1996 Asymmetric hydrogenation of $\beta$ ketophosphonates and $\beta$-ketothiophosphonates with chiral ru (II) catalysts Tetrahedron Lett. 377721

15. Kim D Y and Rhie D Y 1997 Synthesis of $\alpha$ aminoalkylphosphonates from vinylphosphonates via aziridinylphosphonates Tetrahedron 5313603

16. Sawamura M, Ito Y and Hayashi T 1989 Asymmetric synthesis of (1-aminoalkyl) phosphonic acids via asymmetric aldol reaction of (isocyanomethyl) phosphonates catalyzed by a chiral ferrocenylphosphine-gold (I) complex Tetrahedron Lett. 302247

17. Lefebvre I M and Evans S A 1997 Studies toward the asymmetric synthesis of $\alpha$-amino phosphonic acids via the addition of phosphites to enantiopure sulfinimines $J$. Org. Chem. 627532

18. Heydari A, Karimian A and Ipaktschi J 1998 Lithium perchlorate/diethylether catalyzed aminophosphonation of aldehydes Tetrahedron Lett. 396729

19. Fields E K 1952 The Synthesis of Esters of Substituted Amino Phosphonic Acids J. Am. Chem. Soc. 741952

20. Fields E K 1952 The synthesis of esters of substituted amino phosphonic acids ${ }^{1 \mathrm{a}}$ J. Am. Chem. Soc. 741528

21. Ranu B C and Hajra A 2002 A simple and green procedure for the synthesis of $\alpha$-aminophosphonate by a one-pot three-component condensation of carbonyl compound, amine and diethyl phosphite without solvent and catalyst Green Chem. 4551

22. Bhagat S and Chakraborti A K 2008 Zirconium (IV) compounds as efficient catalysts for synthesis of $\alpha$ aminophosphonates J. Org. Chem. 736029

23. Bhagat S and Chakraborti A K 2007 An extremely efficient three-component reaction of aldehydes/ketones, amines, and phosphites (kabachnik- fields reaction) for the synthesis of $\alpha$-aminophosphonates catalyzed by magnesium perchlorate J. Org. Chem. 721263

24. Rezaei Z, Firouzabadi H, Iranpoor N, Ghaderi A, Jafari M R, Jafari A A and Zare H R 2009 Design and 
one-pot synthesis of $\alpha$-aminophosphonates and bis ( $\alpha$ aminophosphonates) by iron (III) chloride and cytotoxic activity Eur. J. Med. Chem. 444266

25. Maghsoodlou M T, Habibi-Khorassani S M, Heydari R, Hazeri N, Sajadikhah S S and Rostamizadeh M 2010 $\mathrm{Al}\left(\mathrm{H} 2 \mathrm{PO}_{4}\right)_{3}$ as an Efficient and Reusable Catalyst for One-pot Three-component Synthesis of $\alpha$-Amino Phosphonates under Solvent-free Conditions Chin. J. Chem. 28285

26. Zhan Z P and Li J P 2005 Bismuth (III) ChlorideCatalyzed Three-Component Coupling: Synthesis of a-Amino Phosphonates Synth. Commun. 352501

27. Ranu B C, Hajra A and Jana U 1999 General procedure for the synthesis of $\alpha$-amino phosphonates from aldehydes and ketones using indium (III) chloride as a catalyst Org. Lett. 11141

28. Xu F, Luo Y, Wu J, Shen Q and Chen H 2006 Facile onepot synthesis of $\alpha$-amino phosphonates using lanthanide chloride as catalyst Heteroatom Chem. 17389

29. Ghosh R, Maiti S, Chakraborty A and Maiti D K 2004 $\mathrm{In}(\mathrm{OTf})_{3}$ catalysed simple one-pot synthesis of $\alpha$-amino phosphonates J. Mol. Catal. A: Chem. 21053

30. Sobhani S and Vafaee A 2009 Efficient one-pot synthesis of $\beta$-hydroxyphosphonates: regioselective nucleophilic ring opening reaction of epoxides with triethyl phosphite catalyzed by $\mathrm{Al}(\mathrm{OTf})_{3}$ Tetrahedron $\mathbf{6 5} 7691$

31. Ghafuri H, Rashidizadeh A and Zand H R E 2016 Highly efficient solvent free synthesis of $\alpha$-aminophosphonates catalyzed by recyclable nano-magnetic sulfated zirconia $\left(\mathrm{Fe}_{3} \mathrm{O}_{4} @ \mathrm{ZrO}_{2} / \mathrm{SO}_{4}^{2-}\right)$ RSC Adv. 616046

32. Kasthuraiah M, Kumar K A, Reddy C S and Reddy C D 2007 Syntheses, spectral property, and antimicrobial activities of 6 - $\alpha$-amino dibenzo $[\mathrm{d}, \mathrm{f}][1,3,2]$ dioxaphosphepin 6-oxides Heteroatom Chem. 182

33. Chandrasekhar S, Prakash S J, Jagadeshwar V and Narsihmulu C 2001 Three component coupling catalyzed by $\mathrm{TaCl}_{5}-\mathrm{SiO}_{2}$ : synthesis of $\alpha$-amino phosphonates Tetrahedron Lett. 425561

34. Wang A, Xu Y, Gao Y, Huang Q, Luo X, An H and Dong J 2015 Chemical and bioactive diversities of the genera Stachybotrys Phytochem. Rev. 14623

35. Pudovik A and Konovalova I 1979 Addition reactions of esters of phosphorus (III) acids with unsaturated systems Synthesis 811979

36. Hou J T, Gao J W and Zhang Z H $2011 \mathrm{NbCl}_{5}$ : An efficient catalyst for one-pot synthesis of $\alpha$ aminophosphonates under solvent-free conditions Appl. Organomet. Chem. 2547

37. Garcia V, Catala-Gregori P, Hernandez F, Megias M and Madrid J 2007 Effect of formic acid and plant extracts on growth, nutrient digestibility, intestine mucosa morphology and meat yield of broilers J. Appl. Poult. Res. 16555

38. Shen R, Chen T, Zhao Y, Qiu R, Zhou Y, Yin S, Wang X, Goto M and Han L-B 2011 Facile regio-and stereoselective hydrometalation of alkynes with a combination of carboxylic acids and group 10 transition metal complexes: selective hydrogenation of alkynes with formic acid J. Am. Chem. Soc. 13317037

39. Soltani O, Ariger M A, Vázquez-Villa $\mathrm{H}$ and Carreira E M 2010 Transfer Hydrogenation in Water: Enantioselective, Catalytic Reduction of $\alpha$-Cyano and $\alpha$-Nitro Substituted Acetophenones Org. Lett. 122893

40. Ariger M A and Carreira E M 2012 pH-Independent transfer hydrogenation in water: Catalytic, enantioselective reduction of $\beta$-keto esters $\mathrm{Org}$. Lett. 144522

41. Ghafuri H and Roshani M 2014 Aqueous formic acid: an efficient, inexpensive and environmentally friendly organocatalyst for three-component Strecker synthesis of $\alpha$-aminonitriles and imines with excellent yields $R S C$ Adv. 458280

42. O’Donnell M J, Lawley L K, Pushpavanam P B, Burger A, Bordwell F and Zhang X-M 1994 Preparation of an $\alpha$-aminophosphonate cation equivalent and its reaction with organoboranes Tetrahedron Lett. 356421

43. Manjula A, Rao V B and Neelakantan P 2003 Onepot synthesis of $\alpha$-aminophosphonates: an inexpensive approach Synth. Commun. 332963

44. Movassagh B and Alapour S 2013 PDodecylbenzenesulfonic Acid: A Highly Efficient Catalyst for One-Pot Synthesis of $\alpha$ Aminophosphonates in Aqueous Media Heteroatom Chem. 24174

45. Bhagat S and Chakraborti A K 2007 An Extremely Efficient Three-Component Reaction of Aldehydes/Ketones, Amines, and Phosphites (Kabachnik-Fields Reaction) for the Synthesis of $\alpha$-Aminophosphonates Catalyzed by Magnesium Perchlorate J. Org. Chem. 721263

46. Zhang X, Qu Y, Fan X, Bores C, Feng D, Andrei G, Snoeck R, De Clercq E, Loiseau M P and Chimiothérapie Antipa 2010 Solvent-free synthesis of pyrimidine nucleoside-aminophosphonate hybrids and their biological activity evaluation Nucleos. Nucleot. Nucl. 29 616

47. Azizi N and Saidi M R 2003 Lithium PerchlorateCatalyzed Three-Component Coupling: A Facile and General Method for the Synthesis of $\alpha$ Aminophosphonates under Solvent-Free Conditions Eur. J. Org. Chem. 20034630 